\title{
Coupled plasmon-phonon mode effects on the Coulomb drag in double-quantum-well systems
}

\author{
K. Güven and B. Tanatar \\ Department of Physics, Bilkent University, Bilkent, 06533 Ankara, Turkey
}

(Received 31 March 1997)

\begin{abstract}
We study the Coulomb drag rate for electrons in a double-quantum-well structure taking into account the electron-optical phonon interactions. The full wave vector and frequency dependent random-phase approximation (RPA) at finite temperature is employed to describe the effective interlayer Coulomb interaction. The electron-electron and electron-optical phonon couplings are treated on an equal footing. The electron-phonon mediated interaction contribution is investigated for different layer separations and layer densities. We find that the drag rate at high temperatures (i.e., $T \geqslant 0.2 E_{F}$ ) is dominated by the coupled plasmon-phonon modes of the system. The peak position of the drag rate is shifted to the low temperatures with a slight increase in magnitude, compared to the uncoupled system results in RPA. This behavior is in qualitative agreement with the recent measurements. Including the local-field effects in an approximate way we also estimate the contribution of intralayer correlations. [S0163-1829(97)05536-7]
\end{abstract}

\section{INTRODUCTION}

Recent advances in the semiconductor processing technology made it possible to manufacture high-quality quantum structures to study various physical effects. In particular, a double-quantum-well system composed of two parallel, spatially separated two-dimensional (2D) electron (or electronhole) gases is well suited to investigate the effects of Coulomb interaction between the carriers in different layers. If the separation distance between the coupled quantum wells is large enough so that the tunneling is insignificant, interesting phenomena stemming from the interlayer correlations arise. When the quantum wells in a double-layer structure are separately contacted the so-called Coulomb drag effect is observed.

The momentum and energy transfer processes between spatially separated electron (and electron-hole) gases were anticipated to influence the transport properties of individual systems because of the Coulomb coupling. ${ }^{1}$ In particular, the Coulomb drag effect, where a current in one layer drives a current in the other one due to the momentum loss caused by interlayer electron-electron interactions, has been observed in various experiments. ${ }^{2-5}$ In these experiments, $2 \mathrm{D}$ electron or hole layers are isolated from each other and the transresistivity in a double-layer system is measured. Theoretical efforts were devoted to the understanding of the observed drag rates and to formulating the framework to understand the many-body aspects of the drag phenomenon. ${ }^{6-10}$ Among the theoretical approaches, there are those based on the Boltzmann transport equation, diagrammatic linear response formalism, and memory function technique. ${ }^{11,12}$ The Coulomb drag effect for quantum wire structures has also attracted some attention, ${ }^{13}$ although no experimental results for these systems have been reported. Recent measurements ${ }^{14-16}$ with an applied magnetic field perpendicular to the coupled layers, offer interesting possibilities in the study of Coulomb drag within the quantum Hall effect regime. ${ }^{17-19}$

The temperature dependence of the observed ${ }^{2,3}$ drag rate (viz., $\tau_{D} \sim T^{2}$ ) supports the identification of Coulomb interactions as the drag mechanism. However, noticeable devia- tions from the $T^{2}$ behavior in the drag rate led Gramila et al. ${ }^{3}$ to suggest that exchange of virtual phonons could be a possible mechanism, since the low-temperature acoustic phonon mean free paths in the used samples were much larger than the layer spacing. Tso and co-workers ${ }^{8}$ have used the momentum balance equations technique to study the effects of virtual phonon exchange on the Coulomb drag, and found that it shows a peak at low temperatures and depends weakly on the separation $d$. They obtained good agreement with experiment when a fitting parameter is used. Zhang and Takahashi ${ }^{20}$ in their calculation of the dynamic conductivity for a double-layer system obtained a stronger separation distance dependence. In these studies, concerned mostly with the low-temperature behavior, the coupling of electrons to the acoustic phonons is considered.

In this paper, we study the enhancement of the Coulomb drag rate due to the coupled plasmon-LO-phonon modes in double-quantum-well systems at zero magnetic field. Since the 2D semiconductor structures widely used in the drag experiments are of polar character (i.e., GaAs), the electronphonon interaction influences most physical properties. We treat the electron-electron and electron-phonon interactions on an equal footing within the random-phase approximation (RPA). Our chief motivation for studying the coupled plasmon-phonon effects in double-quantum-well systems comes from the recent prediction of plasmon enhanced Coulomb drag rate by Flensberg and $\mathrm{Hu}^{9}{ }^{9}$ In contrast to the available experiments ${ }^{2-5}$ performed at low temperatures, they found that the measured momentum transfer rate should exhibit a strong peak at temperatures around $T \sim 0.5 E_{F}$ due to the collective modes of the electronic system. This could provide a new possibility to probe the coupled plasmon modes in double-layer systems. The electron-acoustic phonon interactions play a crucial role in the low-temperature regime. In the high-temperature experiments, just starting to appear, ${ }^{21}$ the electron-optical phonon interactions are likely to be important since they will contribute to the observed drag rate by renormalizing the bare electron-electron interaction. As these many-body effects cannot be disentangled it 


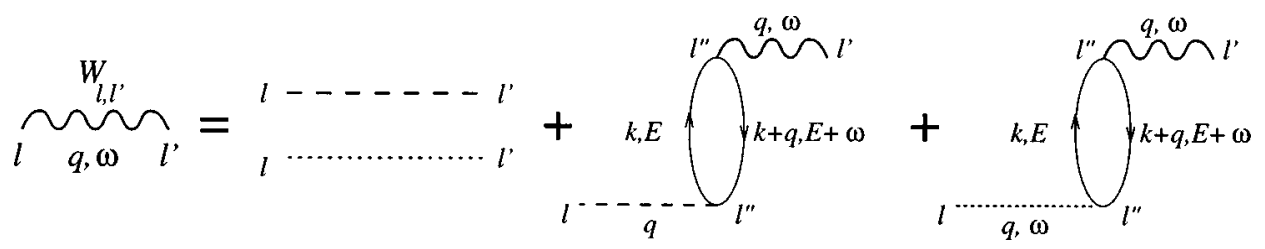

FIG. 1. The diagrammatic representation of the effective interaction $W(q, \omega)$ (denoted by wiggly lines), within the RPA. The dashed and dotted lines stand for the bare Coulomb and unscreened electron-phonon interactions, respectively, whereas the solid lines denote the noninteracting electron propagator defining the polarization (bubble) diagram.

becomes very important to study the role of coupled plasmon-phonon modes for a better understanding of experimental results. We find that the presence of electron-optical phonon coupling modifies the plasmon dispersions, which in turn alters the drag rate than that predicted by the uncoupled RPA results.

In the following, we calculate the temperature dependence of the drag rate between two parallel layers of electrons. Our calculation is mainly based on the random-phase approximation (RPA) which strictly speaking applies only for highdensity systems. We first demonstrate the contribution of the coupled plasmon-phonon modes to the drag rate for $T \geqslant 0.2 E_{F}$. The effects of the coupled electron-phonon system on the layer separation and electronic density dependence of the drag rate are studied. Next, we investigate the influence of the approximate local-field corrections which describe the exchange and correlation effects neglected by the RPA. We find that for realistic systems at the experimentally attainable densities with the present technology such corrections may be quite important.

The rest of this paper is organized as follows. In Sec. II we introduce the formalism by which we calculate the effective interlayer interaction between electrons in a doublequantum-well system. Our results for the drag rate due to coupled plasmon-phonon modes are given in Sec. III. We discuss the influence of layer separation, electron density, and many-body correlations on the temperature dependence of the Coulomb drag rate. We conclude with a brief summary.

\section{MODEL AND THEORY}

We consider two infinite layers of 2D electron gases separated by a distance $d$. It is assumed that the separation distance is large enough to prevent interlayer tunneling, and the possibility of interlayer pairing is neglected. The bare Coulomb interaction between the electrons is given by $V_{i j}(q)=\left(2 \pi e^{2} / \epsilon_{\infty} q\right) e^{-q d\left(1-\delta_{i j}\right)} F_{i j}(q)$, where $F_{i j}(q)$ are the form factors describing the finite width effects ${ }^{7-9}$ of the square wells. They tend to unity for zero-thickness doublelayer system. Note also that we include the high-frequency dielectric constant $\epsilon_{\infty}$, in the Coulomb interaction, as opposed to the usual $\epsilon_{0}$ approximation (static dielectric constant). The areal electron density $N$ in each layer is related to the Fermi wave vector by $N=k_{F}^{2} / 2 \pi$. We also define the dimensionless electron gas parameter $r_{s}=\sqrt{2} /\left(k_{F} a_{B}^{*}\right)$, in which $a_{B}^{*}=\epsilon_{0} /\left(e^{2} m^{*}\right)$ is the effective Bohr radius in the semiconducting layer with background dielectric constant $\epsilon_{0}$ and electron effective mass $m^{*}$. Its numerical value for GaAs is $a_{B}^{*} \approx 100 \AA$. We further assume that only the lowest subband in each layer is occupied, a situation realized in the available experiments. $^{2-5}$

The Coulomb drag rate $\tau_{D}^{-1}$ between the electrons in equal density, double-layer system (to lowest order in the interlayer interaction) has been derived within various theoretical approaches, ${ }^{6-9,12}$ to read

$$
\tau_{D}^{-1}=\frac{1}{8 \pi^{2} m^{*} N T} \int_{0}^{\infty} d q q^{3} \int_{0}^{\infty} d \omega\left|\frac{W_{12}(q, \omega) \operatorname{Im} \chi(q, \omega)}{\sinh (\omega / 2 T)}\right|^{2},
$$

(we take $\hbar$ and $k_{B}$ equal to unity). It gives the rate of momentum transferred from one quantum well to the other. $\chi(q, \omega)$ is density-density response function for electrons in a single layer, and is temperature dependent. In this work, we take $W_{12}(q, \omega)$ to be the dynamically screened effective interaction between electrons in quantum wells 1 and 2, which includes interlayer electron-electron and electron phonon interactions on an equal footing. Thus, the total (effective) electron-electron interaction may be regarded as the sum of a Coulomb term and a contribution arising from the exchange of a virtual LO phonon. Within the RPA, the effective interaction is depicted diagrammatically in Fig. 1. It is obtained by summing the bare Coulomb interaction and phononmediated electron-electron interaction to all orders keeping the "bubble" diagrams. The use of such a restricted set of diagrams (RPA) is justified for high-density (small $r_{s}$ ) electron systems. Furthermore, the weak Fröhlich coupling between the electrons and phonons is well approximated by this perturbative approach. The coupled set of Dyson's equations, generalized ${ }^{20,22-24}$ for a double-layer electron system interacting with bulk, dispersionless LO-phonons of energy $\omega_{\mathrm{LO}}$, yield for the interlayer effective electron-electron interaction

$$
W_{12}(q, \omega)=\frac{V_{12}(q)+\psi_{12}(q, \omega)}{\varepsilon_{\mathrm{TOT}}(q, \omega)}
$$

in which

$$
\psi_{12}(q, \omega)=V_{12}(q)\left[1-\epsilon_{\infty} / \epsilon_{0}\right] \frac{\omega_{\mathrm{LO}}^{2}}{\omega^{2}-\omega_{\mathrm{LO}}^{2}+i \gamma \omega}
$$

is the LO-phonon mediated interlayer electron-electron interaction which depends on wave vector and frequency. Here we have also included a phenomenological lifetime for phonons, i.e., $\gamma^{-1}$. The total screening function for the coupled electron-LO-phonon system is given as

$$
\begin{aligned}
\varepsilon_{\mathrm{TOT}}(q, \omega)= & \left\{1-\left[V_{11}(q)+\psi_{11}(q, \omega)\right] \chi(q, \omega)\right\}^{2} \\
& -\left[V_{12}(q)+\psi_{12}(q, \omega)\right]^{2} \chi^{2}(q, \omega) .
\end{aligned}
$$


In this expression, $\psi_{11}(q, \omega)$ denotes the LO-phonon mediated intralayer electron-electron interaction (defined similar to $\left.\psi_{12}\right)$ and we have made use of the fact that both quantum wells have the same electron density. We relate our expression for the effective interaction to make contact with the previous work ${ }^{8,20}$ in the following. It is customary to write $\varepsilon_{\mathrm{TOT}}(q, \omega)=\varepsilon(q, \omega)-P(q, \omega)$, where $\varepsilon(q, \omega)=[1$ $\left.-V_{11} \chi(q, \omega)\right]^{2}-V_{12}^{2} \chi^{2}(q, \omega)$ is the dielectric function for the double-layer electron system, and $P(q, \omega)$ is the correction due to the electron-phonon coupling, given by

$$
P(q, \omega)=2\left(1-V_{11} \chi\right) \chi \psi_{11}+\chi^{2}\left(\psi_{12}^{2}+2 V_{12} \psi_{12}-\psi_{11}^{2}\right) .
$$

Such a decomposition in single-component systems yields dynamically screened electron-electron interaction and renormalized electron-phonon interaction. ${ }^{22,23}$ In an analogous way, we can write $20,22,24$

$$
W_{12}(q, \omega)=\frac{V_{12}(q)}{\varepsilon(q, \omega)}+\frac{\widetilde{\psi}(q, \omega)}{[\varepsilon(q, \omega)]^{2}},
$$

where

$$
\widetilde{\psi}_{12}(q, \omega)=\frac{\varepsilon \psi_{12}+P V_{12}}{1-P / \varepsilon} .
$$

In the calculation of the drag rate $\tau_{D}^{-1}$, the square of the effective interaction

$$
\begin{aligned}
\left|W_{12}(q, \omega)\right|^{2}= & \frac{\left|V_{12}(q)\right|^{2}}{|\varepsilon(q, \omega)|^{2}}+\frac{2 V_{12}(q)}{|\varepsilon(q, \omega)|^{2}} \operatorname{Re}\left\{\frac{\widetilde{\psi}_{12}}{\varepsilon(q, \omega)}\right\} \\
& +\frac{\left|\widetilde{\psi}_{12}(q, \omega)\right|^{2}}{|\varepsilon(q, \omega)|^{4}},
\end{aligned}
$$

is to be used in Eq. (1). The electron-phonon interaction contribution to the drag rate is determined by the second and the third terms in Eq. (7). Tso et al. ${ }^{8}$ neglected the real phonon-exchange (third term) in their study of electronacoustic phonon coupling and showed that the virtual phonon process (second term) can explain the observed lowtemperature behavior of $\tau_{D}^{-1}$ in double-quantum-well systems. Zhang and Takahashi ${ }^{20}$ argued that the real phononexchange contribution is equally important. In this work we employ Eq. (2) without making any other approximations and calculate $\tau_{D}^{-1}$ for the coupled electron-phonon and uncoupled electron systems (without the electron-phonon interaction). We note that the terminology ${ }^{21}$ used here (and henceforth) refers to coupling of the electron system to phonons. The uncoupled electron system, still has full Coulomb coupling within and between the layers. In both coupled and uncoupled cases we use the high-frequency dielectric constant $\epsilon_{\infty}$ in the bare Coulomb interaction. When phonon coupling is neglected and the static dielectric constant is employed, it is called the $\epsilon_{0}$ approximation. The Coulomb drag rate calculations to date ${ }^{7,9,10,12}$ are performed within the $\epsilon_{0}$ approximation, which includes the lattice effects in the static dielectric constant.

The above expression for the drag rate [Eq. (1)] is derived either using the Boltzmann equation or the diagrammatic perturbation theory ${ }^{9,11}$ and is believed to describe the rel-

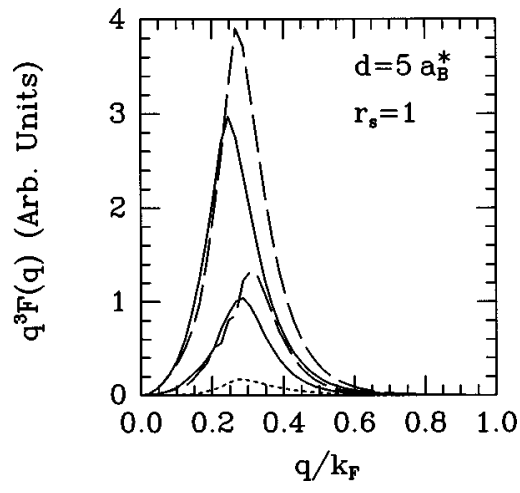

FIG. 2. The integrand of Eq. (8) as a function of $q$ for a doublelayer electron system with $d=5 a_{B}^{*}$ and $r_{s}=1$. The upper and lower curves are at $T=0.5 E_{F}$ and $0.8 E_{F}$, respectively. The solid and dashed lines are for the coupled and uncoupled system, respectively, whereas the dotted line is obtained within the static screening approach (Coulomb interaction only at $T=0.8 E_{F}$ ).

evant experimental situation quite accurately. It was emphasized that the full temperature dependence of the dynamical susceptibility should be used to capture the plasmon contribution at high temperatures. Furthermore, the validity of the above drag rate expression is based ${ }^{9,11}$ on the fact that the intralayer scattering time $\tau(k)$ due to impurities is more or less independent of $k$. Disorder scattering may be accounted for by replacing in Eq. (1) the response function of 2D electron gas $\chi(q, \omega)$, with the susceptibility in the presence of disorder for which one can use the Mermin prescription. ${ }^{25}$

\section{RESULTS AND DISCUSSION}

We use the material parameters appropriate for a $\mathrm{GaAs}$ system for which the recent experiments ${ }^{3-5}$ on drag rate are performed. The high-frequency and static dielectric constants are given, respectively, by $\epsilon_{\infty}=10.9$, and $\epsilon_{0}=12.9$. The LOphonon energy which we take to be dispersionless is $\omega_{\mathrm{LO}}=36.8 \mathrm{meV}$. The phenomenological damping factor is taken in the range $\gamma \approx 0.001-0.1 E_{F}$, and is found not to change the results significantly.

We evaluate the Coulomb drag rate $\tau_{D}^{-1}$ using the effective interaction $W_{12}(q, \omega)$, obtained for a double-layer GaAs system. It contains the direct and electron-phonon mediated electron-electron interactions on an equal footing. We retain the full wave vector, frequency, and temperature dependence in $\varepsilon_{\text {Tот }}(q, \omega)$. In the calculation of the drag rate $\tau_{D}^{-1}$, we need to integrate over the frequencies $\omega$, and wave vector $q$. The frequency dependence of the integrand in Eq. (1) comes from the imaginary parts of the dynamic susceptibilities $\operatorname{Im}[\chi(q, \omega)]$, the thermal factor $\sinh ^{2}(\omega / 2 T)$, and the screening function $\varepsilon_{\mathrm{TOT}}(q, \omega)$ which appears in the denominator. After doing the frequency integral we express $\tau_{D}^{-1}$ as

$$
\tau_{D}^{-1} \sim \int_{0}^{\infty} d q q^{3} F(q),
$$

after similar considerations by Jauho and Smith. ${ }^{7}$ To understand how different screening functions influence the integral, we plot the integrand $q^{3} F(q)$ for two values of the temperature. In Fig. 2, the solid and dashed lines use the dielectric functions appropriate for coupled electron-phonon 


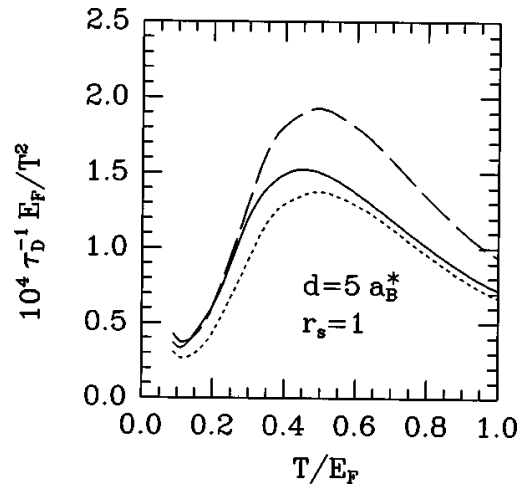

FIG. 3. The scaled drag rate $\tau_{D}^{-1} E_{F} / T^{2}$, as a function of $T$, for a double-layer system with $d=5 a_{B}^{*}$ and $r_{s}=1$. The solid and dashed lines are for the coupled electron-phonon and uncoupled electron systems, respectively, whereas the dotted line is for the uncoupled system within the $\epsilon_{0}$ approximation.

$\left[\varepsilon_{\mathrm{TOT}}(q, \omega)\right]$ and uncoupled electron $[\varepsilon(q, \omega)]$ systems, respectively, keeping the full frequency dependence. We observe that $q^{3} F(q)$ displays a peak around $q \approx 0.2 k_{F}$, and its peak value increases with increasing temperature. The $q$ integral is virtually cutoff at higher momenta because of the factor $e^{-2 q d}$ contained in the function $F(q)$. When the static screening is used for the electronic part, i.e., $\varepsilon(q, \omega=0)$, we obtain a markedly different behavior as shown by the dotted lines in Fig. 2. For the purpose of this illustration, we have ignored the finite well-width effects, taking $F_{i j}(q)=1$, in the bare intralayer and interlayer Coulomb interactions.

The scaled drag rate $\tau_{D}^{-1} / T^{2}$ is shown in Fig. 3 for a double-layer system with $d=5 a_{B}^{*}$ and $r_{s}=1$. We have also assumed here and henceforth the quantum-well thickness to be $L=100 \AA$ A. The results for the coupled and uncoupled systems are denoted by solid and dashed lines, respectively. We observe that within the dynamically screened approach, the drag rate for the coupled and uncoupled systems is very similar at low temperatures. As the temperature increases, the effects of electron-phonon interaction becomes notable. $\tau_{D}^{-1}$ for the coupled electron-phonon system is lower than that of uncoupled electron system. A similar reduction in the scattering rates has been obtained in various many-body calculations ${ }^{22,23}$ of the coupled electron-phonon systems. The difference between the dashed and solid lines provides a direct measure of the extent of phonon coupling. The dotted line in Fig. 3 is the result for uncoupled system within the $\epsilon_{0}$ approximation, where in the bare Coulomb interaction the high-frequency dielectric constant $\epsilon_{\infty}$ is replaced by $\epsilon_{0}$. Thus compared to the previous calculations ${ }^{9,10,12}$ our result for the scaled drag rate $\tau_{D}^{-1} / T^{2}$ has its maximum at a slightly lower temperature, with increased magnitude. In a recent measurement Hill et al. ${ }^{21}$ observed that the drag rate peaks at lower temperatures with a larger maximum than predicted by the RPA results. Our findings for the plasmon-LO-phonon mediated drag are in qualitative agreement with the experiment.

The sudden increase in $\tau_{D}^{-1} / T^{2}$, around $T \sim 0.2 E_{F}$, is due to the thermal excitation of the collective modes in the system. ${ }^{9,12}$ In a double-layer electron system, the zerotemperature optical and acoustic plasmon branches have the long-wavelength behavior given as ${ }^{26,9}$

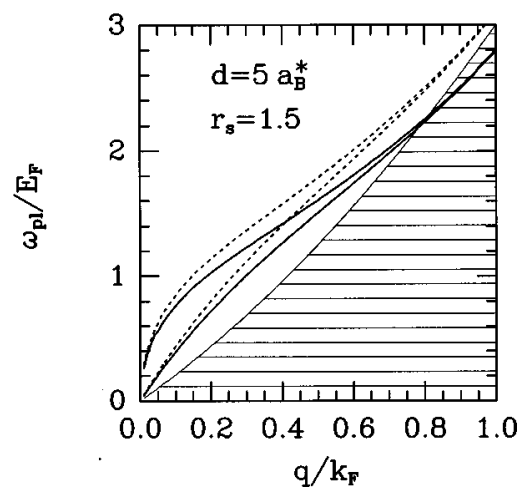

FIG. 4. The plasmon dispersion curves in a double-quantumwell system at $T=0$. The solid and dotted lines show $\omega_{\mathrm{pl}}$ for the coupled and uncoupled systems at $r_{s}=1.5$ and $d=5 a_{B}^{*}$. The hatched region indicates the particle-hole continuum.

$$
\begin{aligned}
\omega_{\mathrm{pl}}^{\mathrm{op}}(q)= & \left(q q_{\mathrm{TF}} k_{F}^{2} / m^{* 2}\right)^{1 / 2} \text { and } \\
& \omega_{\mathrm{pl}}^{\mathrm{ac}}(q)=\frac{q k_{F}}{m^{*}} \frac{1+q_{\mathrm{TF}} d}{\left(1+2 q_{\mathrm{TF}} d\right)^{1 / 2}},
\end{aligned}
$$

respectively, where $q_{\mathrm{TF}}=2 / a_{B}^{*}$ is the Thomas-Fermi screening wave vector in 2D. They refer to the in- and out-of-phase oscillations of the charges in parallel layers. The coupled plasmon-phonon modes can be obtained from the solution of $\operatorname{Re}\left[\varepsilon_{\text {TОт }}\left(q, \omega_{\mathrm{pl}}(q)\right)\right]=0$, when the damping is small. We show the plasmon dispersion relations of a coupled (solid lines) and uncoupled (dotted lines) double-layer system at $T=0$, in Fig. 4. Electron-phonon coupling softens the collective modes, reducing the plasmon energies, and they enter the single-particle excitation region at a finite $q$ value, becoming heavily damped. Thus the acoustic and optical plasmons are more easily excited in a coupled electron-phonon system, and the peak position of $\tau_{D}^{-1} / T^{2}$, moves towards lower temperatures. The uncoupled LO-phonon mode goes over to the TO-phonon mode for small $q$, with energy $\omega_{\text {TO }}$, in the coupled plasmon-phonon system. Its energy remains still quite large to participate in the drag rate.

It has been shown $n^{3,7,8}$ that for low temperatures $\left(T \lesssim 0.2 E_{F}\right)$, when plasmon enhancement is negligible, the drag rate behaves as $\tau_{D}^{-1} \sim d^{-4}$. This is mainly due to the cutoff in the interlayer Coulomb interaction and static screening effects are operative at small frequencies ${ }^{9}$ $\left(\omega \ll q v_{F}\right.$, where $v_{F}$ is the Fermi velocity). In the plasmon dominated regime Flensberg and $\mathrm{Hu}^{9}$ found that $\tau_{D}^{-1}$ approximately has $d^{-3}$ dependence. In Fig. 5, we show $\tau_{D}^{-1} d^{4} / T^{2}$ for different layer separations, for the coupled electron-phonon and uncoupled electron systems. At low $T$, both the coupled and uncoupled drag rates coincide, reflecting the $d^{-4}$ scaling. However, at high temperatures, in the region where collective modes become important, the size of the peak increases with increasing $d$, which indicates a slower falloff than $d^{-4}$. A more systematic study reveals that the peak position for the coupled system moves slightly to lower temperatures as the separation distance is increased.

It is generally believed that the RPA becomes less reliable for electron densities such that $r_{s}>1$ (low density), and even so for low-dimensional systems. In fact, for double-layer 


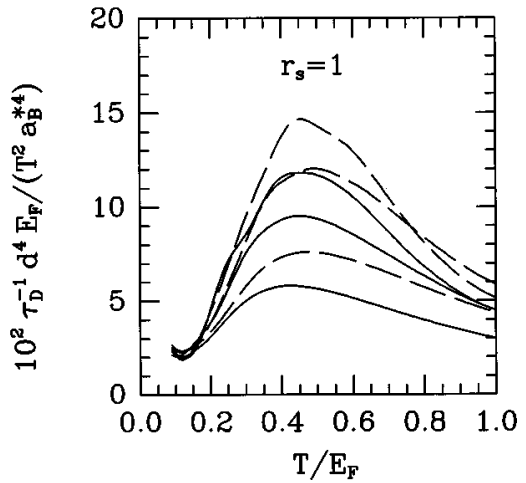

FIG. 5. The scaled drag rate $\tau_{D}^{-1} d^{4} / T^{2}$ as a function of temperature at $r_{s}=1$ for different well separations $d$. The curves from bottom to top indicate $d=3 a_{B}^{*}, 5 a_{B}^{*}$, and $7 a_{B}^{*}$, respectively. The solid and dashed lines are for coupled electron-phonon and uncoupled electron systems, respectively.

electron-hole systems it was found necessary to go beyond the RPA to obtain reasonable agreement with the observed drag rates. ${ }^{10}$ Here we incorporate the correlation effects in an approximate way using local-field corrections. A simplified attempt to go beyond the RPA is provided by the Hubbard approximation in which the Pauli hole around the electrons is taken into account. Neglecting the interlayer correlations but including the intralayer exchange effects (i.e., Hubbard approximation) we take ${ }^{10,12}$

$$
G_{i j}(q)=\frac{1}{2} \frac{q}{\sqrt{q^{2}+k_{F}^{2}}} \delta_{i j},
$$

so that the bare Coulomb interactions are replaced by $V_{i j}(q) \rightarrow V_{i j}(q)\left[1-G_{i j}(q)\right]$ in the screening function $\varepsilon(q, \omega)$. Note that within the Hubbard approximation to the local-field factors, $G_{12}=0$. The interlayer local-field correction should decrease with increasing separation $d$, thus our simple approximation is justified. The calculations by Swierkowski et al. ${ }^{12}$ show that the $G_{12}$ affects the transresistivity in double-layer electron systems very little. The approximate approach of using the Hubbard local-field corrections gives noticeably different results than the RPA. In Fig. 6(a), we show the drag rate with (thick dashed line) and without (thin dashed line, RPA) the local-field corrections.
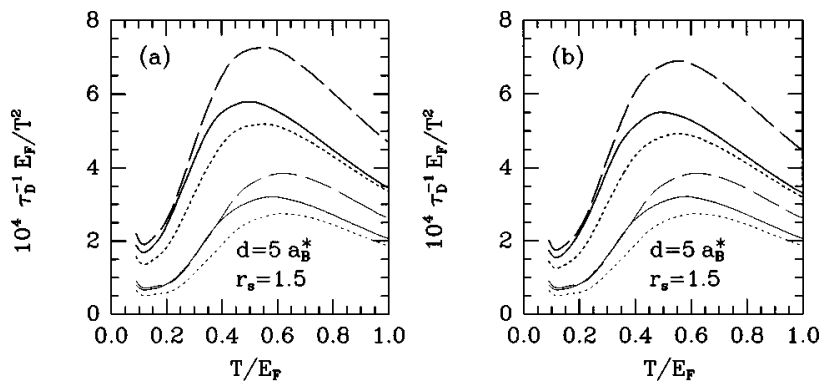

FIG. 6. (a) The scaled drag rate $\tau_{D}^{-1} E_{F} / T^{2}$, as a function of $T$, for a double-layer system with (thick lines) and without (thin lines) the local-field corrections. The solid and dashed curves are for the coupled electron-phonon and uncoupled electron systems, respectively. (b) The same as in (a), except the finite well-width effects are included in the local-field factors.

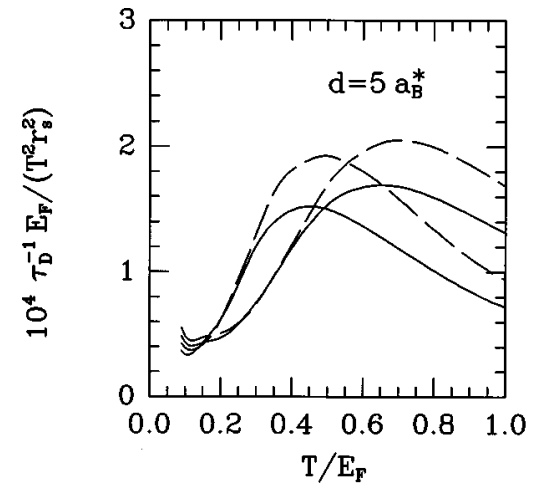

FIG. 7. The scaled drag rate $\tau_{D}^{-1} / T^{2} r_{s}^{2}$ as a function of temperature at $d=5 a_{B}^{*}$ for different layer densities $r_{s}$. The curves from right to left indicate $r_{s}=1$ and $r_{s}=2$, respectively. The solid and dashed lines are for coupled electron-phonon and uncoupled electron systems, respectively.

The presence of correlation effects has a similar influence on the drag rate for the coupled electron-phonon system (shown in Fig. 6 by the thick and thin solid lines). In general, the correlation effects increase the calculated drag rate. ${ }^{12}$ The peak structure in $\tau_{D}^{-1} / T^{2}$ due to plasmon enhancement shifts slightly to lower temperatures. It would be interesting to develop more accurate local-field corrections taking their temperature dependence into account. ${ }^{27}$ However, calculations ${ }^{27}$ of the local-field factor in 3D electron gas, show that the temperature effects become appreciable only for wave vectors $q \gtrsim k_{F}$. Since the region of $q$ integration in Eq. (1) is limited by the factor $\exp (-2 q d)$, it is unlikely that the temperature dependence of $G_{i j}(q)$ in a double-quantum-well system will have a large effect on the calculated drag rates. The Hubbard approximation to the local-field factors given above, as most self-consistent calculations, ${ }^{28}$ does not take the finite width effects into account. Since the effects of finite layer thickness are not negligible in the drag rate, it seems necessary to incorporate them also in the local-field factors. We do this within the present Hubbard approximation to test the quantitative changes brought about by the finite well-width corrected $G_{i j}(q)$. In Fig. 6(b), the finite well-width effects are included in the Hubbard local-field factors, and we observe a slight decrease in the magnitude of the drag rate.

The dependence of the drag rate on electron density parameter $r_{s}$ in each layer is shown in Fig. 7. As the density is decreased, the exchange-correlation effects become more appreciable and $\tau_{D}^{-1}$ is enhanced. The plasmon peak shifts to higher temperatures indicating once again that collective modes are responsible for the observed behavior. We note that the coupled plasmon-phonon mode effects start to deviate from the uncoupled system results at a higher temperature for a lower-density system.

So far, most experiments ${ }^{2-5}$ measuring the Coulomb drag rate in double-layer systems were carried out at low temperatures $\left(T \ll E_{F}\right)$. To observe the plasmon effects suggested by Flensberg and $\mathrm{Hu}^{9}$ the region of high temperatures $T \sim E_{F}$ has to be probed. This has been achieved recently by Hill et al.. ${ }^{21}$ Their transresistivity data indicate a shift towards low temperatures and an enhancement in the magnitude compared to the RPA, $\epsilon_{0}$-approximation calculations. The 
coupled plasmon-LO-phonon mode effects may partially account for the observed discrepancy. Since the RPA is exact only for the high-density regime (i.e., $r_{s} \ll 1$ ), at the experimentally attained densities of $r_{s} \sim 1-1.5$, the correlation effects seem also to be important. ${ }^{12,21}$ More realistic calculations for direct comparison with experiments should take into account the improved local-field corrections ${ }^{12}$ both for the intra and interlayer interactions, perhaps also including the temperature ${ }^{27}$ and well-width dependence of $G_{i j}(q)$.

\section{SUMMARY}

In summary, we have considered the Coulomb drag effect in a double-quantum-well system. The electron-electron and electron-optical phonon interactions are treated on an equal footing with the RPA. The temperature dependence of the drag rate is significantly enhanced when a dynamically screened effective interlayer interaction is used. This enhancement is due to the optical and acoustic plasmons in the double-quantum-well system. The coupling to phonons lowers their energy so that thermal excitation of the coupled modes become favorable at lower temperatures. The localfield effects describing correlations beyond the simple RPA seem to be important for low densities influencing the drag rate considerably.

\section{ACKNOWLEDGMENTS}

This work is partially supported by the Scientific and Technical Research Council of Turkey (TUBITAK) under Grant No. TBAG-AY/123. We thank Dr. M. Z. Gedik, Professor C. M. Sotomayor-Torres, and Dr. N. Balkan for useful discussions.
${ }^{1}$ P. J. Price, Physica B 117B, 750 (1983); M. B. Pogrebinskii, Sov. Phys. Semicond. 11, 372 (1977); P. J. Price, in The Physics of Submicron Semiconductor Devices, edited by H. L. Grubin, D. K. Ferry, and C. Jacoboni (Plenum, New York, 1988).

${ }^{2}$ P. M. Solomon, P. J. Price, D. J. Frank, and D. C. La Tulipe, Phys. Rev. Lett. 63, 2508 (1989).

${ }^{3}$ T. J. Gramila, J. P. Eisenstein, A. H. MacDonald, L. N. Pfeiffer, and K. W. West, Phys. Rev. Lett. 66, 1216 (1991): Phys. Rev. B 47, 12957 (1993); Physica B 197, 442 (1994).

${ }^{4}$ U. Sivan, P. M. Solomon, and H. Shtrikman, Phys. Rev. Lett. 68, 1196 (1992).

${ }^{5}$ H. Rubel, E. H. Linfield, D. A. Ritchie, K. M. Brown, M. Pepper, and G. A. C. Jones, Semicond. Sci. Technol. 10, 1229 (1995).

${ }^{6}$ B. Laikhtman and P. M. Solomon, Phys. Rev. B 41, 9921 (1990); I. I. Boiko and Yu. M. Sirenko, Phys. Status Solidi B 159, 805 (1990).

${ }^{7}$ L. Zheng and A. H. MacDonald, Phys. Rev. B 48, 8203 (1993); A.-P. Jauho and H. Smith, ibid. 47, 4420 (1993).

${ }^{8}$ H. C. Tso, P. Vasilopoulos, and F. M. Peeters, Phys. Rev. Lett. 68, 2516 (1992); 70, 2146 (1993); P. Vasilopoulos and H. C. Tso, in Condensed Matter Theories, edited by L. Blum and F. B. Malik (Plenum, New York, 1993), Vol. 8, p. 81.

${ }^{9}$ K. Flensberg and B. Y.-K. Hu, Phys. Rev. Lett. 73, 3572 (1994); Phys. Rev. B 52, 14796 (1995).

${ }^{10}$ L. Świerkowski, J. Szymański, and Z. W. Gortel, Phys. Rev. Lett. 74, 3245 (1995).

${ }^{11}$ A. Kamenev and Y. Oreg, Phys. Rev. B 52, 7516 (1995); K. Flensberg, B. Y.-K. Hu, A.-P. Jauho, and J. Kinaret, ibid. 52, 14761 (1995).

${ }^{12}$ L. Świerkowski, J. Szymański, and Z. W. Gortel, Surf. Sci. 361/ 362, 130 (1996); Phys. Rev. B 55, 2280 (1997).

${ }^{13}$ Yu. M. Sirenko and P. Vasilopoulos, Superlattices Microstruct. 12, 403 (1992); G. Qin, J. Phys.: Condens. Matter 7, 9785 (1995); B. Tanatar, Solid State Commun. 99, 1 (1996).

${ }^{14}$ N. P. R. Hill, J. T. Nicholls, E. H. Linfield, M. Pepper, D. A. Ritchie, A. R. Hamilton, and G. A. C. Jones, J. Phys.: Condens. Matter 8, L557 (1996).
${ }^{15}$ H. Rubel, A. Fischer, W. Dietsche, K. von Klitzing, and K. Eberl, Phys. Rev. Lett. 78, 1763 (1997).

${ }^{16}$ N. K. Patel, E. H. Linfield, K. M. Brown, M. Pepper, D. A. Ritchie, and G. A. C. Jones, Semicond. Sci. Technol. 12, 309 (1997).

${ }^{17}$ M. C. Bønsager, K. Flensberg, B. Y.-K. Hu, and A.-P. Jauho, Phys. Rev. Lett. 77, 1366 (1996); M. W. Wu, H. L. Cui, and N. J. M. Horing, Mod. Phys. Lett. B 10, 279 (1996); H. C. Tso and P. Vasilopoulos, Phys. Rev. B 45, 1333 (1992); G. Qin, Solid State Commun. 101, 267 (1997).

${ }^{18}$ Y. B. Kim and A. J. Millis (unpublished); I. Ussishkin and A. Stern (unpublished).

${ }^{19}$ E. Shimshoni and S. L. Sondhi, Phys. Rev. B 49, 11484 (1994); D. Orgad and S. Levit, ibid. 53, 7964 (1996).

${ }^{20}$ C. Zhang and Y. Takahashi, J. Phys.: Condens. Matter 5, 5009 (1993).

${ }^{21}$ N. P. R. Hill, J. T. Nicholls, E. H. Linfield, M. Pepper, D. A. Ritchie, G. A. C. Jones, B. Y.-K. Hu, and K. Flensberg, Phys. Rev. Lett. 78, 2204 (1997).

${ }^{22}$ R. Jalabert and S. Das Sarma, Phys. Rev. B 40, 9723 (1989); S. Das Sarma and B. A. Mason, Ann. Phys. (N.Y.) 163, 78 (1985); S. Das Sarma, J. K. Jain, and R. Jalabert, Phys. Rev. B 37, 6290 (1988).

${ }^{23}$ N. Tzoar and C. Zhang, Phys. Rev. B 35, 7596 (1987).

${ }^{24}$ B. A. Sanborn, Phys. Rev. B 51, 14256 (1995).

${ }^{25}$ N. D. Mermin, Phys. Rev. B 1, 2362 (1970).

${ }^{26}$ S. Das Sarma and A. Madhukar, Phys. Rev. B 23, 805 (1981); G. E. Santoro and G. F. Giuliani, ibid. 37, 937 (1988).

${ }^{27}$ S. Tanaka and S. Ichimaru, J. Phys. Soc. Jpn. 55, 2278 (1986); H. Schweng, H. M. Böhm, A. Schinner, and W. Macke, Phys. Rev. B 44, 13291 (1991).

${ }^{28}$ K. I. Golden and De-xin Lu, Phys. Rev. A 45, 1084 (1992); J. Szymański, L. Świerkowski, and D. Neilson, Phys. Rev. B 50, 11002 (1994); L. Zheng and A. H. MacDonald, ibid. 49, 5522 (1994). 